\title{
Validation of the Chinese version of the Coma Recovery Scale-Revised (CRS-R)
}

Ying Zhang, Jing Wang, Caroline Schnakers, Minhui He, Hong Luo, Lijuan Cheng, Fuyan Wang, Yunzhi Nie, Wangshan Huang, Xiaohua Hu, Steven Laureys \& Haibo Di

To cite this article: Ying Zhang, Jing Wang, Caroline Schnakers, Minhui He, Hong Luo, Lijuan Cheng, Fuyan Wang, Yunzhi Nie, Wangshan Huang, Xiaohua Hu, Steven Laureys \& Haibo Di (2019) Validation of the Chinese version of the Coma Recovery Scale-Revised (CRS-R), Brain Injury, 33:4, 529-533, DOI: 10.1080/02699052.2019.1566832

To link to this article: https://doi.org/10.1080/02699052.2019.1566832

View supplementary material $\smile$

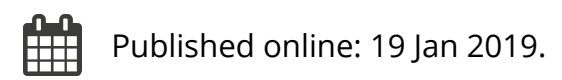

Submit your article to this journal $\widetilde{ }$

III Article views: 85

Q View related articles $\sqsubset$

View Crossmark data $\nearrow$

约

Citing articles: 1 View citing articles ¿ 


\title{
Validation of the Chinese version of the Coma Recovery Scale-Revised (CRS-R)
}

\author{
Ying Zhang ${ }^{a, b}$, Jing Wang $\mathbb{1}^{* a}$, Caroline Schnakers*,d, Minhui He ${ }^{a, b}$, Hong Luo ${ }^{b}$, Lijuan Cheng ${ }^{a, e}$, Fuyan Wang ${ }^{a, b}$, \\ Yunzhi Nie ${ }^{\mathrm{a}, \mathrm{f}}$, Wangshan Huang ${ }^{\mathrm{a}}$, Xiaohua Hu${ }^{\mathrm{g}}$, Steven Laureys ${ }^{\mathrm{h}}$, and Haibo $\mathrm{Di}^{\mathrm{a}}$
}

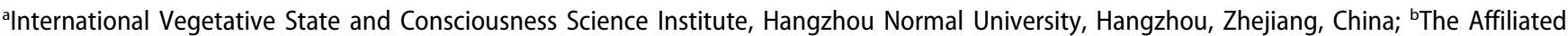
Hospital of Hangzhou Normal University, Hangzhou Normal University, Hangzhou, Zhejiang, China; 'Research Institute, Casa Colina Hospital and

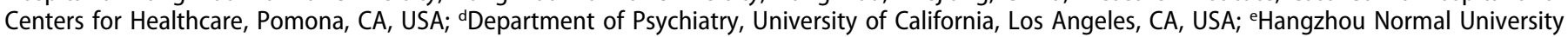
Qianjiang College, Hangzhou, Zhejiang, China; ${ }^{f}$ Ningbo NO.7 Hospital, Ningbo, Zhejiang, China; ${ }^{9}$ Department of Rehabilitation, Hangzhou Wujing

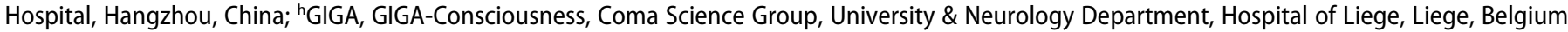

\begin{abstract}
Primary Objective: This study aims to validate the Chinese version of the Coma Recovery Scale-Revised (CRS-R).

Methods: One hundred sixty-nine patients were assessed with both the CRS-R and the Glasgow Coma Scale (GCS), diagnosed as being in unresponsive wakefulness syndrome (UWS, formerly known as vegetative state), minimally conscious state (MCS), or emergence from MCS (EMCS). A subgroup of 50 patients has been assessed twice by the same rater, within $24 \mathrm{~h}$. Patient outcome was documented six months after assessment.

Results: The internal consistency for the CRS-R total score was excellent (Cronbach's $a=0.84)$. Good test-retest reliability was obtained for CRS-R total score and subscale scores (intra-class correlation coefficient $[\mathrm{ICC}]=0.87$ and ICC $=0.66-0.84$, respectively). Inter-rater reliability was high $(\mathrm{ICC}=0.719$; $p<0.01)$. Concurrent validity was good between CRS-R total scale and GCS total scale. Diagnostic validity was excellent compared with GCS (emerged from UWS: $24 \%$; emerged from MCS: $28 \%$ ). When considering patient outcome, diagnostic validity was good. In addition, false-positive rates have been detected for both diagnoses.

Conclusion: The Chinese version of the CRS-R is a reliable and sensitive tool and can discriminate patients in UWS, MCS, and EMCS successfully.
\end{abstract}

\section{ARTICLE HISTORY}

Received 28 January 2018

Revised 15 November 2018

Accepted 5 January 2019

\section{KEYWORDS}

Behavioral scale; Coma Recovery Scale-Revised; consciousness; unresponsive wakefulness syndrome; minimally conscious state

\section{Background}

Differentiating reflex from voluntary activity is one of the most challenging tasks facing clinicians involved in the care of patients with severe brain injury, i.e., disorders of consciousness (DOC), such as unresponsive wakefulness syndrome (UWS) or minimally conscious state (MCS) $(1,2)$. Behavioral assessment is currently considered as the main way to detect signs of consciousness and, hence, the main way to determine diagnosis.

However, behavioral assessment is complicated by the presence of motor impairments, tracheostomy, high fluctuations in vigilance, or ambiguous and rapidly extinguished responses. Because of these compromising factors, accurate diagnosis can rapidly become difficult to make. Previous studies have shown that around $40 \%$ of the patients diagnosed as being in vegetative state (VS) are misdiagnosed and are in fact conscious (3-6). As misdiagnosis can lead to serious consequences, especially, in terms of rehabilitation strategies, pain treatment, and end-of-life decision-making (7), sensitive valid tools have to be used to assess remaining cognitive functioning linked to consciousness and to quantify the severity of DOC. The Glasgow Coma Scale
(GCS) is among of the scales the mostly used in China to assess patients with severe brain injuries, but its appropriateness to assess signs of consciousness has previously been debated $(8,9)$. Coma Recovery Scale-Revised (CRS-R) was originally developed by investigators from the JFK Johnson Rehabilitation Institute in 1991 to improve and facilitate the differential diagnosis between UWS and MCS (10). A revised version of this scale was published in 2004 (11). In 2010, a report of the American Congress of Rehabilitation Medicine aiming to provide a systematic review of behavioral assessment scales for DOC has indicated that, when compared to other scales (including the GCS), the CRS$\mathrm{R}$ meets the highest psychometric standards for the evaluation of the even minimally reserved consciousness in patients with several brain injuries (8). According to some studies, CRS-R is a more sensitive scale than other scales that were previously developed to evaluate DOC patients $(9,12)$. However, that report mentions few evidences or studies with small sample sizes for some aspects of its reliability and validity such as its internal consistency and its test-retest reliability. The report also states that the diagnostic validity of the CRS-R remains unproven. The CRS-R has been translated into English, French, Spanish, Italian,

CONTACT Haibo Di dihaibo19@aliyun.com International Vegetative State and Consciousness Science Institute, Hangzhou Normal University, Hangzhou 310036

*These authors contributed equally to the manuscript.

Clinical trials registration: ClinicalTrials.gov identifier NCT03126929

(6) Supplementary data can be accessed here.

(c) 2019 Taylor \& Francis Group, LLC 
Norwegian, Portuguese, and Polish (9,13-17). In order to promote the development of the clinical and research fields on DOC in China, CRS-R has been translated into Chinese and has been published (18). Hence, this study aims to assess the internal consistency, the test-retest reliability, inter-rater reliability, concurrent validity, and the diagnostic sensitivity of the Chinese version of the CRS-R in a large sample of DOC patients.

\section{Methods}

\section{Participants}

Patients were recruited from the intensive care unit, the neurology unit, and the rehabilitation unit of the University Hospital of Hangzhou (Zhejiang, China).

One hundred sixty-nine patients have been included in our study. The inclusion criteria were (1) adults, (2) pre-screening performed by a referring physician confirming the presence of a disorder of consciousness in accordance with international diagnostic criteria for UWS, MCS, or emergence from MCS (EMCS) $(1,2),(3)$ native Chinese language, and (4) good hearing. Exclusion criteria were (1) neuromuscular blocking agents or sedative drugs administered within the prior $24 \mathrm{~h}$, (2) documented history of a prior coma, critical illness, or unstable medical condition, and (3) superior limb contusions or fractures.

The causes of brain injury were traumatic $(n=73)$, hemorrhagic $(n=68)$, hypoxic ischemic $(n=18)$, cerebral tumor $(n=4)$, and others $(n=6)$. Seventy patients were in an acute stage (less than 28 days after injury) and 99 were in a subacute to chronic stage (more than 28 days after injury). The age of all patients in this study ranged from 18 to 86 years old. Our sample included 117 males and 52 females (see Table 1).

The study was approved by the Ethical Committee of the University Hospital of Hangzhou. The patients' relatives and caregivers were informed about the experimental procedure and signed a written informed consent. The study was conducted according to the World Medical Association's Declaration of Helsinki.

\section{Procedure}

The Chinese version of the CRS-R was administered (see Supplemental Material) (18). The CRS-R consists of 23 items grouped in six subscales addressing auditory, visual, motor, oromotor, communication, and arousal functions. The higher items represent conscious-related behavior, while the lower

Table 1. Demographic characteristics of patients.

\begin{tabular}{lcr}
\hline Characteristics & Median & Range \\
\hline Age (years) & 58 & $18-86$ \\
& $n$ & \\
Days post-injury & 70 & $1-28$ \\
Acute & 99 & $>28$ \\
Non-acute & & \\
Gender & 117 & \\
Males & 52 & \\
Females & & \\
Etiology & 73 & \\
TBI & 96 & \\
NTBI &
\end{tabular}

TBI: traumatic brain injury; NTBI: non-traumatic brain injury. items for each subscale represent reflexive activity. The basis for scoring was the presence or absence of the specific behavior in response to standard stimuli.

The CRS-R was administered to 169 patients by an experienced rater (initially trained by the Coma Science Group, Belgium). Total scores were recorded to investigate the internal consistency (which measures the extent to which scale items are related to the same general construct and produce similar ratings), and subscale scores were scored to investigate the internal correlations of CRS-R. Test-retest reliability indicates that the consistency between the two results obtained from two different tests demonstrated the stability of the CRS-R scale. In order to test it, a subgroup of 50 patients was assessed twice (only using the CRS-R) by the same rater, on two consecutive days. Besides, CRS-R was administered to the same 50 patients by another rater within $24 \mathrm{~h}$ on day 1 . Each rater recorded patients' responses independently during the assessment process. Patient was diagnosed as UWS, MCS or EMCS based on the CRS-R assessment. To assess diagnostic validity and concurrent validity, CRS-R and GCS were administered in a randomized order by an experienced rater to assess patients' consciousness. The GCS scale includes three subscales that address arousal level (eye opening), motor function, and verbal abilities. Subscale scores are summed and yield a total score ranging from 3 (worst) to 15 (best). Both scales (i.e., GCS and CRS-R) have been administered in our entire sample $(n=169)$ during the same assessment. Based on the diagnostic criteria of the Multi-Society Task Force (for the VS) (1) and of the Aspen workgroup (for the MCS) (2), operational definitions have been developed using the items represented on each scale (Table 2). For each patient, a diagnosis has been derived using the operationally defined cutoff scores for UWS, MCS, and EMCS described in Table 2. The numbers of diagnostic agreement and disagreement between scales have been reported. The patient outcome has also been documented six months after the assessments and categorized as UWS, MCS, or EMCS using these international diagnostic criteria $(1,2)$.

\section{Statistical analysis}

Descriptive statistics for patients are presented as means \pm SD or medians with interquartile ranges. A Cronbach's alpha $(\alpha)$ is known as an internal consistency estimate of the reliability of

Table 2. Diagnostic criteria for the VS, the MCS, and the EMCS for each scale (CRS-R and GCS).

\begin{tabular}{llll}
\hline $\begin{array}{l}\text { Behavioural } \\
\text { scale }\end{array}$ & \multicolumn{1}{c}{ VS } & \multicolumn{1}{c}{ MCS } & \multicolumn{1}{c}{ EMCS } \\
\hline CRS-R & Auditory $\leq 2$ AND & Auditory $=3-4$ OR & Motor $=6$ OR \\
& Visual $\leq 1 A N D$ & Visual $=2-5$ OR & Communication \\
& Motor $\leq 2$ AND & Motor $=3-5$ OR & $=2$ \\
& Oromotor $/$ Verbal $\leq 2$ & Oromotor $/$ & \\
& $A N D$ & Verbal $=3$ OR & \\
& Communication $=0$ & Communication $=1$ & \\
& $A N D$ & & \\
GCS & Arousal $\leq 2$ & & \\
& Eyes opening $=2-4$ & Verbal $=3-4$ OR & Verbal $=5$ \\
& $A N D$ & Motor $=5-6$ & \\
& Verbal $=1-2$ AND & & \\
& Motor $=1-4$ & &
\end{tabular}

CRS-R: Coma Recovery Scale-Revised; GCS: Glasgow Coma Scale; VS: vegetative state; MCS: minimally conscious state; EMCS: emergence from minimally conscious state. 
test scores, which generally increases as the intercorrelations among test items increase. a was used to calculate the internal consistency of total scores of the CRS-R and internal correlations of the subscale scores by the Spearman rank correlation method. It assesses how well the relationship between the two variables. A coefficient greater than or equal to 0.81 is typically considered excellent, between 0.61 and 0.80 is considered good, between 0.4 and 0.6 is considered fair-to-moderate, and values of 0.4 or less are considered poor results (19). Test-retest reliability for total score of the CRS-R and for its subscale scores was evaluated by an intra-class correlation coefficient (ICC). ICC is a descriptive statistic that can be used as quantitative measurements of units that are organized into groups. Thresholds for the ICC values have been defined as follows: high (ICC > 0.90), good (ICC: 0.60-0.90), and low $($ ICC < 0.60) (13). Inter-rater reliability was determined by an ICC. For all patients, concurrent validity was computed by Spearman rank correlation method between CRS-R total score and GCS total score. Diagnostic validity was assessed using chi-squares (threshold for significance at $p<0.05$ ) on the proportion of misdiagnosis (UWS vs. MCS) according to the time since injury (acute vs. non-acute) or the etiology (traumatic vs. non-traumatic).

\section{Results}

\section{Internal consistency}

The Cronbach's alpha was 0.84 for total scores of all patients' CRS-R assessment, which suggests a high degree internal consistency of Chinese version of CRS-R. In addition, Spearman test values referring to intercorrelations among the subscales scores are reported in Table 3.

\section{Test-retest reliability}

The ICC between CRS-R total scores obtained on two occasions was $0.87(p<0.001)$, which indicated that the scale is relatively stable when used on different occasions. The coefficients obtained for CRS-R subscale scores were $0.74(p<0.001)$ for the auditory subscale, $0.82(p<0.001)$ for the visual subscale, $0.84(p<0.001)$ for the motor subscale, $0.77(p<0.001)$ for the verbal subscale, $0.83(p<0.001)$ for the communication subscale, and $0.66(p<0.001)$ for the arousal subscale.

\section{Inter-rater reliability}

Inter-rater reliability for the CRS-R total score was high (ICC $=0.719 ; p<0.01)$, which indicates that CRS-R could yield reproducible findings across raters during the evaluation process.
Table 4. Spearman coefficient between CRS-R total score and total score of GCS scale in acute stage, in non-acute stage, and in both stages.

\begin{tabular}{lrcc}
\hline & $N$ & Spearman coefficient $(r)$ & $p$ \\
\hline Both stages & 169 & 0.78 & $<0.01$ \\
Acute stage & 70 & 0.86 & $<0.01$ \\
Non-acute stage & 99 & 0.69 & $<0.01$ \\
\hline
\end{tabular}

\section{Concurrent validity}

Total scores of the CRS-R were correlated with the GCS total scores to compute concurrent validity $(n=169)$. Spearman coefficient was significant between the CRS-R scale and GCS scale for the total sample $(r=0.78, p<0.01, n=169)$ as well as also when considering different time injury (i.e., acute: $r=0.86, p<0.01, n=70$; non-acute: $r=0.69, p<0.01$, $n=99$; see Table 4).

\section{Diagnostic validity}

Each patient was assigned a diagnosis of UWS, MCS, or EMCS following completion of the CRS-R. In 15 out of 169 patients assessed, it produced a diagnosis of EMCS based on the CRS-R, 63 patients received a diagnosis of MCS, and 91 patients diagnosed as UWS based on the CRS-R (see Table 5).

Chi-squared analysis showed that the proportion of the patients diagnosed with EMCS and MCS by the CRS-R was significantly higher when compared to the GCS $\left(\chi^{2}=9.139\right.$, $p<0.05)$. Moreover, CRS-R could identify 28 patients as being in MCS who were otherwise misclassified as being in UWS by the GCS (i.e., misdiagnosis rate of 24\%) and 14 patients as being EMCS who were misclassified as being in MCS by the GCS (i.e., misdiagnosis rate of $28 \%$ ). For the MCS diagnosis, behaviors that the most frequently missed for subscales included: automatic motor response $(n=12)$; visual pursuit and fixation $(n=9)$; consistent/reproducible movement to command $(n=4)$; and non-functional communication $(n=4)$. For the EMCS diagnosis, the missed behaviors were functional object use $(n=11)$ and functional communication $(n=9)$ (see Table 6). One MCS patient, nevertheless, was classified as being in UWS by the CRS-R, while the GCS

Table 5. Frequency of diagnosis for VS, MCS, and EMCS between GCS and CRS-R diagnoses $(n=169)$.

\begin{tabular}{rlrccr}
\hline & \multicolumn{5}{c}{ GCS } \\
\cline { 3 - 6 } & & VS & MCS & EMCS & Total \\
\hline \multirow{3}{*}{ CRS-R } & VS & 90 & 1 & 0 & 91 \\
& MCS & 28 & 35 & 0 & 63 \\
& EMCS & 0 & 14 & 1 & 15 \\
& Total & 118 & 50 & 1 & 169 \\
\hline
\end{tabular}

CRS-R: Coma Recovery Scale-Revised; GCS: Glasgow Coma Scale; VS: vegetative state; MCS: minimally conscious state; EMCS: emergence from minimally conscious state.

Table 3. Subscale score intercorrelations of the Chinese version of the Coma Recovery Scale-Revised (CRS-R).

\begin{tabular}{lccccc}
\hline & Auditory & Visual & Motor & Oromotor/verbal & Communication \\
\hline Auditory & 1.000 & & & & \\
Visual & 0.874 & 1.000 & & & \\
Motor & 0.636 & 0.649 & 1.000 & 1.000 & \\
Oromotor/verbal & 0.371 & 0.337 & 0.367 & 0.320 & 1.000 \\
Communication & 0.782 & 0.749 & 0.569 & 0.150 & 0.515 \\
Arousal & 0.482 & 0.525 & 0.323 & 1.000 \\
\hline
\end{tabular}


Table 6. Behavioral signs of consciousness found using CRS-R in patients misdiagnosed as being in VS or MCS.

\begin{tabular}{llc}
\hline Subscale & \multicolumn{1}{c}{ Behaviour } & $\begin{array}{c}\text { Number of } \\
\text { observation }\end{array}$ \\
\hline Auditory & Consistent movement to command & 3 \\
& $\begin{array}{l}\text { Reproducible movement to } \\
\text { command }\end{array}$ & 1 \\
Visual & Object recognition & 3 \\
& Object localization & 2 \\
& Visual pursuit & 5 \\
Motor & Fixation & 4 \\
& Functional object use & 11 \\
& Automatic motor response & 12 \\
Verbal & Object manipulation & 7 \\
Communication & Localization to noxious stimulation & 2 \\
Arousal & Functional: accurate & 0 \\
& Non-functional: intentional & 9 \\
& Attention & 4 \\
\end{tabular}

classified him as being in MCS (i.e., misdiagnosis rate of 1\%). The CRS-R has been unable to detect the presence of localization to pain. No significant differences in misdiagnosis rate were found according to the time since injury $\left(\chi^{2}=0.05\right.$, $p=0.82)$ or the etiology $\left(\chi^{2}=0.43, p=0.51\right)$.

Using international diagnostic criteria $(1,2)$, the patient outcome has been documented six months after the assessment in 25 of the 28 patients misdiagnosed as being in UWS and in 7 of the 14 patients misdiagnosed as being in MCS. Twenty-one of 25 patients (84\%) were still in MCS as initially diagnosed by the CRS-R (the four others were in UWS), while 6 of 7 patients (86\%) were still in EMCS (one was in MCS) as initially diagnosed by the CRS-R, suggesting a low falsepositive rate for both diagnoses (i.e., $16 \%$ and $14 \%$, respectively). The patient misdiagnosed by the CRS-R was in UWS, six months after the assessment.

\section{Discussion}

The essential aim of this study was to validate the Chinese version of the CRS-R in a large sample of patients with DOC. Results showed that the translated Chinese version has a good internal consistency, test-retest reliability, inter-rater agreement as well as a better diagnostic validity as compared to the GCS, indicating that Chinese version is a reliable and valid scale to access patients' responsiveness.

In our large dataset $(n=169)$, the internal consistency of the scale was high $(\alpha=0.84)$, which supports a previous preliminary study obtained in a smaller sample size $(n=80)$ $(\alpha=0.83)$ (11). The Chinese version of the CRS-R seems, therefore, a reliable homogeneous neuro-behavioral measure for assessing consciousness. Test-retest reliability for total score as well as for all subscale scores was also good, suggesting that the CRS- $\mathrm{R}$ is a pretty stable scale when used within an interval of $24 \mathrm{~h}$. This finding is parallel to the initial study (11) as well as to two recent studies in which the reliability of the scale was tested in fewer patients $(14,16)$. In addition, in this study, an inter-rater agreement for the total score was high (ICC $=0.719 ; p<0.01$ ), which was close to the study validated the French version of the scale $(k=0.80)(9)$.

The Chinese version of the CRS- $\mathrm{R}$ has been able to detect signs of consciousness in $24 \%$ of the patients who would have been diagnosed as being in UWS using the GCS, which demonstrated significantly higher diagnostic sensitivity in detecting MCS and EMCS patients compared to the GCS. This finding relates to one previous smaller sample study (9). Besides, two other studies compared the diagnostic sensitivity of the CRS-R to a consensus-based diagnosis $(4,12)$. In both studies, the CRS-R allowed detecting more conscious patients. The most frequently missed conscious behaviors were spontaneous automatic motor responses, visual pursuit, and fixation. Both behaviors are not included in the GCS which explains why they were not detected. Visual pursuit and fixation have been shown as one of the first signs of consciousness recovered after severe brain injury (20) but have also been identified as the most difficult behaviour to detect in DOC patients $(4,6,9)$, confirming the importance to assess carefully this sign of consciousness using sensitive scales such as the CRS-R $(21,22)$. Our study also showed that the CRS$\mathrm{R}$ scale allows a better diagnosis in EMCS patients than using GCS scale. Even though both scales assess functional communication (i.e., highest score of the verbal subscale of the GCS and highest score of the communication subscale of the CRS-R), this behavior has been missed in 9 of 14 misdiagnosed patients, suggesting that the CRS-R assesses such behavior in a more sensitive way. Moreover, six months after the assessment, the majority of those misdiagnosed patients (more than 80\%) were under the same level of consciousness, which suggested a low level of false positives. On the contrary, the MCS patient misdiagnosed as UWS by the CRS-R was found to be a false positive as his 6-month outcome was a UWS state (and not MCS as previously determined by the GCS). Finally, misdiagnosis did not seem to differ according to the time since injury and etiology. All those evidences suggest that the CRS-R has a very good diagnostic validity whoever is the patient assessed (i.e., UWS or MCS, acute or subacute to chronic, traumatic, or nontraumatic).

\section{Limitations}

The study has, nevertheless, several limitations. First, there was no comparison of the diagnosis of nurses, caregivers, and physiotherapists in this study. So, the comparison between them is necessary for the future research. Second, when testing DOC patients, behavioral responses can vary widely from an assessment to another due to the fluctuations of the patient's level of vigilance. Even though our patients have been assessed twice within $24 \mathrm{~h}$, it is difficult to fully exclude this bias. Patients' fluctuation of vigilance could explain why the arousal subscale had the lowest test-retest agreement. It is, therefore, likely that the test-retest reliability of the CRS-R is higher than what we observed. From these, we found that multiple assessments in the diagnosis of DOC patients are important. Third, the diagnostic validity could be measured differently. In this study, we chose to compare the diagnosis obtained using the CRS-R to another standardized behavioral scale widely used in China (i.e., the GCS) as well as to estimate the amounts of false positives using 6-month follow-up and published criteria for DOC diagnosis $(1,2)$. However, Wessex Head Injury Matrix (WHIM) is a higher sensitive scale relative to the GCS. To determine what is a gold standard is really difficult in DOC patients. Therefore, we will compare the CRS-R with the 
WHIM. We also want to compare the diagnosis obtained using the CRS-R to the diagnosis obtained using a consensus agreement between two or more expert clinicians relying on bedside observation. Various methodologies leading to the same results would be an additional argument to demonstrate the diagnostic sensitivity of the CRS-R.

\section{Conclusions}

Our results confirm that the Chinese version of the CRS-R is a valid and reliable tool and that this scale should be used systematically when assessing the level of consciousness of patients with DOC.

\section{Funding}

This work was supported by the National Natural Science Foundation of China under Grant 81471100; the National High Technology Research and Development Program of China (863 Program) under Grant 2015AA020514; the Hangzhou Normal University under Grant PD11002010002016; the Research Fund for International Young Scientists of the National Natural Science Foundation of China under Grant 811247008; the Medical Science and Technology Projects in Zhejiang Province under Grants 2013KYB213 and 2015KYA181; and the College students in Zhejiang Province Science and Technology Innovation Activities plan under Grant 2017R423055.

\section{Acknowledgments}

We greatly appreciate the statistical advices provided by Jiqian Fang.

\section{Disclosure of interest}

The authors report no conflict of interest.

\section{ORCID}

Jing Wang (D) http://orcid.org/0000-0003-2445-4966

\section{References}

1. Multi-Society Task Force on PVS. Medical aspects of the persistent vegetative state (2). N Engl J Med. 1994;330(22):1572-79. doi:10.1056/NEJM199406023302206.

2. Giacino JT, Ashwal S, Childs N, Cranford R, Jennett B, Katz DI, Kelly JP, Rosenberg JH, Whyte J, Zafonte RD, et al. The minimally conscious state: definition and diagnostic criteria. Neurology. 2002;58(3):349-53.

3. van Erp WS, Lavrijsen JC, Vos PE, Bor H, Laureys S, Koopmans RT. The vegetative state: prevalence, misdiagnosis, and treatment limitations. J Am Med Dir Assoc. 2015;16(1):85.e9-e14. doi:10.1016/j.jamda.2014.10.014.

4. Schnakers C, Vanhaudenhuyse A, Giacino J, Ventura M, Boly M, Majerus S, Moonen G, Laureys S. Diagnostic accuracy of the vegetative and minimally conscious state: clinical consensus versus standardized neurobehavioral assessment. BMC Neurol. 2009;9(1):35. doi:10.1186/1471-2377-9-35.

5. Andrews K, Murphy L, Munday R, Littlewood C. Misdiagnosis of the vegetative state: retrospective study in a rehabilitation unit. BMJ. 1996;313(7048):13-16.
6. Childs NL, Mercer WN, Childs HW. Accuracy of diagnosis of persistent vegetative state. Neurology. 1993;43(8):1465-67.

7. Andrews K. Medical decision making in the vegetative state: withdrawal of nutrition and hydration. NeuroRehabilitation. 2004; 19(4):299-304.

8. American Congress of Rehabilitation Medicine BI-ISIGDoCTF, Seel RT, Sherer M, Whyte J, Katz DI, Giacino JT, Rosenbaum AM, Hammond FM, Kalmar K, Pape TL, et al. Assessment scales for disorders of consciousness: evidence-based recommendations for clinical practice and research. Arch Phys Med Rehabil. 2010; 91(12):1795-813. doi:10.1016/j.apmr.2010.07.218.

9. Schnakers C, Majerus S, Giacino J, Vanhaudenhuyse A, Bruno MA, Boly M, Moonen G, Damas P, Lambermont B, Lamy M, et al. A French validation study of the Coma Recovery Scale-Revised (CRS-R). Brain Inj. 2008;22(10):786-92. doi:10.1080/02699050802403557.

10. Giacino JT, Kezmarsky MA, DeLuca J, Cicerone KD. Monitoring rate of recovery to predict outcome in minimally responsive patients. Arch Phys Med Rehabil. 1991;72(11):897-901.

11. Giacino JT, Kalmar K, Whyte J. The JFK Coma Recovery Scale-Revised: measurement characteristics and diagnostic utility. Arch Phys Med Rehabil. 2004;85(12):2020-29.

12. Estraneo A, Moretta P, De Tanti A, Gatta G, Giacino JT, Trojano L, Italian Crs RMVG. An Italian multicentre validation study of the Coma Recovery Scale-Revised. Eur J Phys Rehabil Med. 2015;51(5):627-34.

13. Tamashiro M, Rivas ME, Ron M, Salierno F, Dalera M, Olmos L. A Spanish validation of the Coma Recovery Scale-Revised (CRS-R). Brain Inj. 2014;28(13-14):1744-47. doi:10.3109/ 02699052.2014.947621.

14. Sacco S, Altobelli E, Pistarini C, Cerone D, Cazzulani B, Carolei A. Validation of the Italian version of the Coma Recovery Scale-Revised (CRS-R). Brain Inj. 2011;25(5):488-95. doi:10.3109/ 02699052.2011.558043.

15. Lovstad M, Froslie KF, Giacino JT, Skandsen T, Anke A, Schanke AK. Reliability and diagnostic characteristics of the JFK Coma Recovery Scale-Revised: exploring the influence of rater's level of experience. J Head Trauma Rehabil. 2010;25(5):349-56. doi:10.1097/HTR.0b013e3181cec841.

16. Simoes JFFL, Jesus LMT, Voegeli D, Sa-Couto P, Fernandes J, Morgado M. Assessment of comatose patients: a Portuguese instrument based on the Coma Recovery Scale-Revised and using nursing standard terminology. J Adv Nurs. 2011; 67(5):1129-41. doi:10.1111/j.1365-2648.2010.05559.x.

17. Binder M, Gorska U, Wojcik-Krzemien A, Gociewicz K. A validation of the Polish version of the Coma Recovery Scale-Revised (CRSR). Brain Inj. 2018;32(2):242-46. doi:10.1080/ 02699052.2017.1406991.

18. Di H, He M, Zhang Y, Cheng L, Wang F, Nie Y, Huang W, Laureys S, Schnakers C. Chinese translation of the Coma Recovery Scale-Revised. Brain Inj. 2017;31(3):363-65. doi:10.1080/ 02699052.2016.1255780.

19. Shrout PE. Measurement reliability and agreement in psychiatry. Stat Methods Med Res. 1998;7(3):301-17. doi:10.1177/ 096228029800700306.

20. Dolce G, Lucca LF, Candelieri A, Rogano S, Pignolo L, Sannita WG. Visual pursuit in the severe disorder of consciousness. J Neurotrauma. 2011;28(7):1149-54. doi:10.1089/neu.2010.1405.

21. Vanhaudenhuyse A, Schnakers C, Bredart S, Laureys S. Assessment of visual pursuit in post-comatose states: use a mirror. J Neurol Neurosur Psychiatry. 2008;79(2):223. doi:10.1136/jnnp.2007.121624.

22. Wannez S, Vanhaudenhuyse A, Laureys S, Bredart S. Mirror efficiency in the assessment of visual pursuit in patients in minimally conscious state. Brain Inj. 2017;31(11):1429-35. doi:10.1080/02699052.2017.1376755. 